\title{
AN OSCILLATION CONDITION FOR DIFFERENTIAL EQUATIONS OF ARBITRARY ORDER
}

\author{
WILLIAM F. TRENCH
}

\begin{abstract}
In separate papers, D. L. Lovelady has related oscillation of solutions of certain linear differential equations of odd order $>3$ and even order $>4$ to oscillation of an associated second order equation. This paper presents a unified proof of Lovelady's results for equations of arbitrary order $>3$. The results are somewhat more detailed and the equations need not be linear.
\end{abstract}

D. L. Lovelady [2], [3] has proved the following theorem.

THEOREM 1. Suppose $p$ is positive and continuous on $[0, \infty)$, and

$$
\int^{\infty} p(s) s^{n-3} d s<\infty
$$

where $n>2$. Suppose also that the equation

$$
w^{\prime \prime}+\left(\frac{1}{(n-3) !} \int_{t}^{\infty}(s-t)^{n-3} p(s) d s\right) w=0 \quad(t>0)
$$

is oscillatory. Then every solution of

$$
y^{(n)}+p(t) y=0 \quad(t>0)
$$

is oscillatory if $n$ is even, while if $n$ is odd and $y$ is nonoscillatory, then $y^{\prime}, \ldots, y^{(n-1)}$ tend monotonically to zero and $y$ approaches a finite limit as $t \rightarrow \infty$.

Lovelady gave separate proofs of the two halves of this theorem, considering even $n$ in [3] and odd $n$ in [2]. In this paper we adapt his methods to study oscillation of solutions of

$$
y^{(n)}+f(t, y)=0 \quad(t>0)
$$

under the following assumptions.

Assumption A. Let $n$ be any integer $>3$ and let $f$ be continuous on $(0, \infty) \times$ $(-\infty, \infty)$, with

$$
f(t, y) / y>p(t)>0 \quad(y \neq 0)
$$

where $p$ is nonnegative and continuous on $(0, \infty)$ and satisfies (1).

As usual, we define an extendible solution of (3) to be one which exists on some interval $(T, \infty)$. Such a solution is said to be oscillatory if it has a zero on every interval $\left(T_{1}, \infty\right)$. Because of (4), a nonoscillatory solution $y$ of (3) must satisfy the

Received by the editors March 3, 1980; presented to the Society, April 18, 1980.

AMS (MOS) subject classifications (1970). Primary 34C10, 34C11.

Key words and phrases. Oscillation. 
inequality $y(t) y^{(n)}(t)<0$ for sufficiently large $t$. The following elementary lemma of Kneser [1] describes the possible asymptotic behavior of such functions.

Lemma 1. Suppose $y(t)>0$ and $y^{(n)}(t)<0$ for large $t$. Then

(a) If $n$ is even, there is an even integer $k$ such that

$$
\begin{gathered}
y^{(j)}(t)>0 \quad \text { for } t>t_{0}, 0<j<k+1, \\
\lim _{t \rightarrow \infty} y^{(k+1)}(t)=\alpha \quad(\text { finite } \geq 0),
\end{gathered}
$$

and

$$
\lim _{t \rightarrow \infty} y^{(j)}(t)=0, \quad k+2<j<n-1 .
$$

(b) If $n$ is odd, then either there is an odd integer $k$ for which (5), (6), and (7) hold, or

$$
\lim _{t \rightarrow \infty} y^{(j)}(t)=0, \quad 1<j \leqslant n-1
$$

and

$$
\left.\lim _{t \rightarrow \infty} y(t)=\gamma \quad \text { (finite }>0\right) \text {. }
$$

The limits in (6), (7), (8), and (9) are all approached monotonically for large $t$. Moreover, these assertions remain valid if the stated inequalities on $y, y^{\prime}, \ldots, y^{(n)}$ and $\alpha$ and $\gamma$ are all reversed.

For convenience, we define

$$
q(t)=\int_{t}^{\infty}(t-s)^{n-3} p(s) d s
$$

which exists, because of (1).

The proofs of the next two theorems are adaptations of Lovelady's proofs.

ThEOREM 2. Suppose Assumption A holds and $k$ is an integer such that $n-k$ is even and $0<k<n-4$. Suppose also that the equation

$$
w^{\prime \prime}+\frac{q(t)}{(n-k-3) ! k !} w=0 \quad(t>0)
$$

is oscillatory. Then no nonoscillatory solution of (3) can satisfy either (5), (6), and (7) as stated, or (5), (6), and (7) with the inequalities on $y^{(7)}$ and $\alpha$ reversed.

Proof. We will show that if (3) has an eventually positive solution $y$ which satisfies (5) and (7), then (10) is nonoscillatory. From (3) and (7),

$$
y^{(k+2)}(t)=\frac{1}{(n-k-3) !} \int_{t}^{\infty}(t-s)^{n-k-3} f(s, y(s)) d s .
$$

Therefore,

$$
-y^{(k+2)}(t)>\frac{1}{(n-k-3) !} \int_{t}^{\infty}(s-t)^{n-k-3} p(s) y(s) d s \quad\left(t>t_{0}\right)
$$


because of (4), (5), and the assumption that $n-k$ is even. If $k>1$, then (5) implies that

$$
y(s)>\frac{1}{(k-1) !} \int_{t_{0}}^{s}(s-\lambda)^{k-1} y^{(k)}(\lambda) d \lambda \quad\left(t>t_{0}\right) .
$$

Substituting this into (11) yields

$$
\begin{aligned}
-y^{(k+2)}(t) \geqslant & \frac{1}{(n-k-3) !(k-1) !} \\
& \cdot \int_{t}^{\infty}(s-t)^{n-k-3} p(s) \int_{t}^{s}(s-\lambda)^{k-1} y^{(k)}(\lambda) d \lambda d s \quad\left(t>t_{0}\right) .
\end{aligned}
$$

Since $y^{(k+1)}(t) \geqslant 0$ if $t \geqslant t_{0}$ (see (5)), $y^{(k)}(\lambda)>y^{(k)}(t)$ if $\lambda \geqslant t>t_{0}$; hence, (13) implies that

$$
-y^{(k+2)}(t)>\frac{q(t)}{(n-k-3) ! k !} y^{(k)}(t) \quad\left(t>t_{0}\right) .
$$

Although we assumed that $k \geqslant 1$ in deriving this inequality, it can be seen from (11) that it also holds for $k=0$.

Now define $v(t)=y^{(k+1)}(t) / y^{(k)}(t)$; then

$$
v^{\prime}(t)+v^{2}(t)=\frac{y^{(k+2)}(t)}{y^{(k)}(t)}<\frac{-q(t)}{(n-k-3) ! k !} \quad\left(t>t_{0}\right) .
$$

By a theorem of Wintner [5] (see also [4, p. 63]), the existence of a continuously differentiable function $v$ which satisfies the inequality in (14) implies that (10) is nonoscillatory. This completes the proof in the case where $y(t)>0$ for large $t$. Because of (4), the proof for the case where $y(t)<0$ for large $t$ is similar.

TheOrem 3. Suppose Assumption A holds and $k$ is an integer such that $n-k$ is even and $1 \leqslant k \leqslant n-2$. Suppose also that the equation

$$
w^{\prime \prime}+\frac{q(t)}{(n-k-2) !(k-1) !} w=0 \quad(t>0)
$$

is oscillatory. Then the conclusion of Theorem 2 holds.

Proof. Again we suppose that $y$ is an eventually positive solution of (3) which satisfies (5) and (7). From (3), (6), and (7),

$$
y^{(k+1)}(t) \geqslant \frac{1}{(n-k-2) !} \int_{t}^{\infty}(t-s)^{n-k-2} f(s, y(s)) d s \quad\left(t>t_{0}\right) .
$$

Because of (4) and the assumption that $n-k$ is even, this implies that

$$
y^{(k+1)}(t)>\frac{1}{(n-k-2) !} \int_{t}^{\infty}(s-t)^{n-k-2} p(s) y(s) d s \quad\left(t>t_{0}\right) .
$$

From this and (12),

$$
\begin{aligned}
y^{(k+1)}(t) \geqslant & \frac{1}{(n-k-2) !(k-1) !} \\
& \cdot \int_{t}^{\infty}(s-t)^{n-k-2} p(s) \int_{t}^{s}(s-\lambda)^{k-1} y^{(k)}(\lambda) d \lambda d s \quad\left(t>t_{0}\right) .
\end{aligned}
$$


Now define a sequence $\left\{u_{m}\right\}$ of functions on $\left[t_{0}, \infty\right)$ by $u_{0}(t)=y^{(k)}(t)$ and, for $m \geqslant 1$,

$$
\begin{aligned}
u_{m}^{\prime}(t)= & \frac{1}{(n-k-2) !(k-1) !} \\
& \cdot \int_{t}^{\infty}(s-t)^{n-k-2} p(s) \int_{t}^{s}(s-\lambda)^{k-1} u_{m-1}(\lambda) d \lambda d s \quad\left(t>t_{0}\right), \\
u_{m}\left(t_{0}\right)=y^{(k)}\left(t_{0}\right) &
\end{aligned}
$$

By induction,

$$
y^{(k)}\left(t_{0}\right) \leqslant u_{m+1}(t) \leqslant u_{m}(t) \leqslant y^{(k)}(t) \quad\left(t>t_{0}, m>0\right)
$$

and

$$
0<u_{m+1}^{\prime}(t)<u_{m}^{\prime}(t)<y^{(k+1)}(t) \quad\left(t>t_{00} m>0\right)
$$

Therefore, $\left\{u_{m}\right\}$ is uniformly bounded and equicontinuous on finite subintervals of $\left[t_{0}, \infty\right)$, so the Arzela-Ascoli theorem and the monotonicity with respect to $m$ imply that $u(t)=\lim _{m \rightarrow \infty} u_{m}(t)$ exists on $\left[t_{0}, \infty\right)$, that the convergence is uniform on finite subintervals, and that $u$ is continuous. From (18), $\left\{u_{m}^{\prime}\right\}$ also converges on $\left[t_{0}, \infty\right)$; moreover, it can be shown that the integrals in (16) converge uniformly on finite subintervals of $\left[t_{0}, \infty\right)$. Therefore, $u$ is differentiable and

$$
\begin{aligned}
u^{\prime}(t)= & \frac{1}{(n-k-2) !(k-1) !} \\
& \cdot \int_{t}^{\infty}(s-t)^{n-k-2} p(s) \int_{t}^{s}(s-\lambda)^{k-1} u(\lambda) d \lambda d s \quad\left(t>t_{0}\right) .
\end{aligned}
$$

If $k=n-2$, differentiating this yields

$$
u^{\prime \prime}(t)+\frac{q(t)}{(n-3) !} u(t)=0 \quad\left(t>t_{0}\right)
$$

Since

$$
u(t) \geqslant y^{(k)}\left(t_{0}\right)>0 \quad\left(t>t_{0}\right)
$$

from (17), we have now shown that (15) (with $k=n-2$ ) has a nonoscillatory solution, which contradicts our assumption and completes the proof for this case. If $1<k<n-2$, differentiating (19) yields

$$
u^{\prime \prime}(t)+\frac{q(t)}{(n-k-2) !(k-1) !} u(t)=P(t) \quad\left(t>t_{0}\right)
$$

with

$$
P(t)=\frac{-1}{(n-k-3) !(k-1) !} \int_{t}^{\infty}(s-t)^{n-k-3} p(s) \int_{t}^{s}(s-\lambda)^{k-1} u(\lambda) d \lambda d s<0 .
$$

Thus, $u(t)>0$ on $\left[t_{0}, \infty\right)$ and the left side of (20) is negative. We will show that this contradicts our assumption that (15) is oscillatory. 
Let $t_{1}$ and $t_{2}$ be successive zeros of a nontrivial solution $w$ of (15), with $t_{0}<t_{1}<t_{2}$, and assume without loss of generality that

$$
w(t)>0 \quad\left(t_{1}<t<t_{2}\right) .
$$

Then

$$
w^{\prime}\left(t_{1}\right)>0, \quad w^{\prime}\left(t_{2}\right)<0 .
$$

If $W=u w^{\prime}-u^{\prime} w$, then (22) implies that

$$
W\left(t_{1}\right)=u\left(t_{1}\right) w^{\prime}\left(t_{1}\right)>0 \text { and } W\left(t_{2}\right)=u\left(t_{2}\right) w^{\prime}\left(t_{2}\right)<0 .
$$

However, from (15), (20), and (21),

$$
W^{\prime}(t)=-P(t) w(t)>0 \quad\left(t_{1}<t<t_{2}\right),
$$

which contradicts (23). This completes the proof.

If $1<k \leqslant n-4$, then Sturm's comparison theorem implies that Theorem 3 is stronger than Theorem 2 when $2 k<n-2$, and that the opposite is true when $2 k>n-2$.

The following theorem is our main result.

Theorem 4. Suppose Assumption A holds and (2) is oscillatory. Then (a) if $n$ is even, every extendible solution of (3) is oscillatory; (b) if $n$ is odd and $y$ is an extendible nonoscillatory solution of $(3)$, then $y^{\prime}, \ldots, y^{(n-1)}$ tend monotonically to zero as $t \rightarrow \infty$, and $\lim _{t \rightarrow \infty} y(t)=\gamma$ (finite). In this case, $\gamma=0$ if

$$
\int^{\infty} s^{n-1} p(s) d s=\infty \text {. }
$$

Proof. Since $m ! n !<(m+n)$ ! for any positive integers $m$ and $n$, Sturm's comparison theorem and the assumption that (2) is oscillatory imply that (10) and (15) are oscillatory for every admissible $k$. Therefore, Lemma 1 and Theorems 2 and 3 imply all the conclusions of this theorem except that $\gamma=0$ if (24) holds. To see the latter, note that if $y$ is a solution of (3) which satisfies (8) and (9), then

$$
y(t)=\gamma+\frac{1}{(n-1) !} \int_{t}^{\infty}(s-t)^{n-1} f(s, y(s)) d s .
$$

If $\gamma>0$, then $f(s, y(s)) \geqslant \gamma p(s)$ for large $s$, so the existence of the integral in (25) contradicts (24). The conclusion follows in a similar way if $\gamma<0$. This completes the proof.

\section{REFERENCES}

1. A. Kneser, Untersuchungen über die reelen Nullstellen der Integrale linearer Differentialgleichungen, Math. Ann. 42 (1893), 409-435.

2. D. L. Lovelady, An asymptotic analysis of an odd order linear differential equation, Pacific J. Math. 57 (1975), 475-480.

3. Oscillation and even order linear differential equations, Rocky Mountain J. Math. 6 (1976), 299-304.

4. C. A. Swanson, Comparison and oscillation theory of linear differential equations, Academic Press, New York, 1968.

5. A. Wintner, On the non-existence of conjugate points, Amer. J. Math. 73 (1951), 368-380.

Department of Mathematical Sciences, Drexel University, Philadelphia, Pennsylvania 19104 